\title{
ON THE CHANGES
}

WHICH OCCUR IN

\section{BONE AND SOFT TISSUES AFTER AMPUTATION OF A LIMB,}

GEORGE POLLOCK, F.R.C.S., CONSULTING SURGKON TO ST. GEORGR'S HOSPITAL.

Received December 8th, 1885-Read February 23rd, 1886.

THE changes which take place in bone after amputation of a portion of a limb present some interesting features, and are, I have ventured to think, of sufficient importance pathologically, and perhaps to some extent practically, to render the subject worthy of consideration by the Fellows of the Society.

The subject is not a new one. Some of the changes to which attention will be drawn have been remarked on already, but certain other conditions do not appear to have been particularly noticed; and it is this which has led me to hope that a discussion of the subject will not be considered useless or wasteful of time. 
The changes referred to are not, however, confined exclusively to bone tissue; to some extent they affect the softer structures.

They are not only found to occur in the bone of a stump of an amputated limb, but also in limbs or parts affected by paralysis. But changes in a marked degree will also be observed, though in a different form and due to a different cause, in bones of parts which have to undergo, or take upon themselves, an extra amount of work, to compensate for the loss of other parts with which they were originally associated and had to act.

My attention was first drawn to this subject by an opportunity afforded me of examining the body of a very old man, who many years previously had undergone amputation of one leg, a short distance above the knee, and had evidently long survived the operation. The subject had been received in the dissecting room of St. George's Hospital ; no history could be obtained as to the cause of the amputation, or as to the date of the operation, nor of the subsequent occupation of the individual. Suffice it to say that the stump was well healed and sound, and the cicatrix was evidently of considerable age.

The observations, therefore, as regards this individual case, are simply confined to the description of the more interesting points exemplified in the specimens of bone figured on Plate IX. These consist of the upper portions of two thigh-bones from the same subject, with the head, neck, and great trochanter complete in each. For the illustrations of these specimens I am indebted to my friend Mr. John H. Morgan, Assistant Surgeon to Charing Cross Hospital. The characteristics of the two specimens are accurately represented, and the differences between the bone of the amputated leg and that of the entire femur made very clear.

To indicate accurately the comparative changes illustrated in the drawings, and the specimens themselves, the thigh-bone of the sound side has been sawn through, at a point to make it correspond in length with that of the 
amputated side, measured from the upper edge of the great trochanter.

It will be observed, on an examination of the specimens, that the contrast between them is most marked. The general appearance, the thickness, weight, obliquity of neck, and the respective positions of the head of each femur,-all these points tell without trouble which portion of femur must have been taken from the amputated limb, and which belonged to the sound side.

The difference in weight of the two bones is very marked. That taken from the sound side weighed 6 oz. gr. xx. The corresponding portion from the stump weighed 3 oz. ziij.

The difference in the obliquity of the neck of the femur of the two sides which occurs after an amputation of a limb through the thigh-bone, is perhaps one of the most interesting and prominent features to be noted, so far as the bone is affected by conditions entirely dependent on, and occurring subsequent to, the loss of a leg above the knee.

It has been found, from the examination of many specimens, that if the subject has lived some few years after an amputation through the thigh, the neck of the mutilated femur will become by degrees very oblique. In the specimen exhibited this is seen to have taken place to a remarkable extent : in contrast to this the neck of the femur of the perfect bone has gradually been brought down to a right angle with the shaft, and lies horizontally between the head and the trochanter. The head of the femur on the side of operation, as compared with the upper edge of the trochanter, is nearly an inch higher than in the opposite limb. The shaft in one is thin and light in weight. The shaft of the other is thickened, hardened, and increased in size beyond its natural growth; more in character with that of a femur of middle age than of one taken from the body of a man eighty years of age.

A most interesting contrast is thus exhibited in these 
two specimens. Not only is the neck of the femur of the amputated side seen to be extremely oblique, but that of the sound limb has not only assumed the horizontal position, but the bone itself, neck and shaft, has become thickened, strengthened, and hardened. The extra weight imposed on the sound limb by the amputation of the opposite one, to a great extent, no doubt, assisted to produce this alteration in the neck; the necessary extra muscular action of the sound limb and consequent increased blood-supply was most probably the chief cause of the addition to the substance of the bone. It will thus occur that the increasing obliquity of the neck of the femur on the amputated side gradually adds to the length of the remaining portion of the bone; consequently, for some time after an amputation has been performed, there is a tendency for the stump to become gradually more and more conical, unless precautions have been taken to obviate such an occurrence by the removal of a sufficient portion of the shaft, a fact which should not be lost sight of in the performance of amputation through the thigh.

On the other hand, it will be found that the height of the individual who has undergone amputation through the thigh will diminish to a slight extent, from the circumstance that the neck of the femur on the sound side gradually yields to the extra pressure from above until it has assumed the horizontal position.

The increased obliquity of the neck of the femur after amputation through the thigh is probably due to more than one cause. The removal of the natural weight of the trunk from the head of the bone may exert some influence ; but probably more may be due to the fact that the bone is no longer supported from below, but is suspended, as it were, from the cotyloid cavity; it may also be partly owing to the daily decreasing support from the surrounding muscles of the stump. The deterioration observed to take place in the bone after an amputation of the thigh is not, however, limited to that portion of the bone left to form the stump. Similar conditions of marked 
diminished nutrition, and consequent wasting of structure, are found to extend their influence to the pelvis of the amputated side. Specimens of this condition have been kindly brought for exhibition by Dr. Humphry, of Cambridge, and Mr. Howard Marsh.

Dr. Humphry was able to secure a specimen of the pelvis with the stump and perfect thigh-bone, from a subject in which he had amputated through the piddle of the femur some years previously. In this instance, the evident loss of substance of the pelvis, on the side corresponding to the mutilated femur, can be at once detected; so marked is it, that no one could hesitate, without examination of the thigh-bones, to pronounce on which side the amputation had been performed.

In the specimen exhibited to the meeting by $\mathrm{Mr}$. Howard Marsh, similar conditions to those seen in the preparation from the Cambridge Museum are to be observed. It shows a diminution in size and thinning on the side which corresponds to the amputated thigh. The history of the case is not recorded. The specimen is from St. Bartholomew's Hospital.

In a living subject who has undergone amputation through the femur certain alterations may be detected, such as are borne out by the examination of these parts after death. I had the opportunity of examining a case under the care of Mr. Henry Morris, in Middlesex Hospital, of which the following are the brief particulars :

W. W-, æt. 49, had had his left leg amputated about the junction of the middle with the lower third of the femur, for disease of the knee-joint, when about ten years of age. The following were the measurements of the respective parts :-

On the amputated side, the measurement round the upper part of the thigh was twenty. and a half inches, that on the sound side was twenty-two inches. The measurement of the right half of the pelvis, from the median line of the sacrum behind, to the linea alba in front, was fifteen inches. The corresponding measurement of the opposite side was four- 
teen inches and a half. From the anterior superior spine of ilium on the sound side to the middle line of symphysis pubis was six inches and an eighth, while that of the opposite side was only five inches and five eighths.

The trochanter of the amputated limb was much less prominent than that of the perfect extremity and could not be very readily distinguished. It was on a lower level than that of the entire femur. In another case in Middlesex Hospital the patient had had his leg amputated for disease of the knee-joint, some four years previously. The measurement from the median line behind to the linea alba in front on the amputated side, was twelve inches and five eighths. That on the sound side was thirteen inches.

I am not aware that attention has been drawn to the occurrence of this alteration in the aspect and conditions of the pelvis, following on amputation of the thigh. Mr. Hilton $^{1}$ some years ago drew attention to a somewhat similar alteration of the pelvis following on hip-joint disease in children, though I cannot find that any allusion is made to the changes referred to, which occur after amputation. He writes: "I have ascertained by examination that the os innominatum on the side of the disease does not grow so rapidly, and finally is not so large as its fellow; hence the area of the pelvis is not symmetrical, and thus may interfere with parturition at the full period of gestation. I may add that this pelvic deformity is most conspicuous when the hip disease occurs in, or continues into, the period of early menstruation."

The deformity here described is, however, to be alone attributed to some arrest of growth, whereas that which takes place after an amputation of the thigh, may occur after growth has ceased, and then can alone be the result of absorption of bone tissue, the partial result probably of diminished action of all muscles attached to that portion of the pelvis.

Similar conditions of wasted or wasting bone structure are to be observed under other circumstances, but all bearing

$$
1 \text { 'Rest and Pain,' 2nd edit., p. } 320 .
$$


on the same principle, and illustrative of the fact that wherever there is diminished action there is reduced nutrition, and wherever we find extra action there will be found increased growth.

Loss of substance of bone occurs in many conditions of disease ; the wasting of the jaws when all teeth have been parted with, as often seen in advanced life; thinning of bone under conditions of infantile paralysis ; or that which is attendant on anchylosis of a joint ; all these conditions manifestly indicate a diminished blood supply and diminished nutrition followed by a gradual absorption of bony tissue.

To illustrate somewhat practically and more precisely some of the conditions to which the foregoing remarks apply, I cannot do better than quote Mr. Hilton's observations when referring to these changes in a case of disease of the shoulder-joint: "The anchylosis and its remote effects manifested themselves in this way: the humerus and scapula were dwarfed and moved rigidly together, and, in addition to the rigidity of that joint, the clavicle was short, as compared with the other side, and the chest on the left or shoulder-disease side was not so much developed as on the other; hence the left lung and chest-wall were not in true concord as a part of the respiratory apparatus" (loc. cit., p. 319).

Through the kindness of Mr. Henry Morris I am able to exhibit a very interesting specimen of the wasting of bone in association with paralysis. The preparation consists of the bones of a right upper extremity with scapula and clavicle, all showing extreme atrophy; all the bones are very light and fragile. The shaft of the humerus is not thicker than a fibula and is twisted. The radius and ulna are rounded and about equal in diameter to a goose-quill. Both extremities of the humerus and lower end of the radius have been fractured. The case was no doubt that of an adult, as all the epiphyses are ossified to the shafts of their respective bones.

We not only may observe these changes as taking 
place in bones, but the soft tissues, such as muscle, \&c., are equally influenced as regards waste or increase under similar circumstances. The waste or increase of muscle may be observed under many conditions.

In the case of a patient the subject of an amputation through the femur we may detect both the one and the other slowly progressing side by side. On the amputated side we find wasting of muscle consequent on diminished muscular action, and lessened blood supply; on the sound side, the substance of the thigh is found to have increased in bulk and the muscles have become largely developed.

A gentleman under the care of Mr. Henry Morris, was the subject of popliteal aneurism of the left leg. The right leg had been amputated by Mr. Nunn eight years previously for ruptured popliteal aneurism. I was requested by Mr. Morris to see the case in consultation with him late one evening. For reasons which need not be entered into it was decided that the femoral artery should be tied without delay. The operation was most successfully performed by Mr. Morris the following morning.

The left thigh had become very stout and muscular, the patient having made constant active use of it, with the aid of crutches and an artificial leg.

When examining the case before the operation, it was found difficult to command the circulation through the aneurism without using considerable pressure over the femoral just below Poupart's ligament. This difficulty was evidently occasioned by the quantity of soft tissue between the skin and the artery, and this accumulation of fat rendered treatment by pressure of the artery out of the question. The amount of pressure necessary to command the circulation would most certainly have shortly produced slough or ulceration of the skin.

The artery was tied in Scarpa's triangle. When the sartorius muscle was drawn to one side its increased size was a very marked object of attention; its breadth being quite twice that of the usual size of this muscle. Similar 
enlargement was observed in the deeper muscles, so that the femoral sheath lay deeper than I had ever previously observed it.

Mr. Morris kindly allowed me to take measurements of the respective sides a few days after the performance of the operation. On the sound side the measurement round the thigh close to the groin was 23 inches and $\frac{10}{16}$ ths. On the amputated side the measurement round the stump equally near the groin was 21 inches and $\frac{11}{16}$ ths, a difference of nearly two inches.

The trochanter on the amputated side was lower than that of the perfect leg, but its outline could not be very clearly defined.

Sir Benjamin Brodie, to whose teaching I owe not a little, drew attention to the wasting of bones when limited in their natural movements. He writes, "You will observe that all bones in a state of inaction lose a great part of their phosphate of lime."l I cannot, however, find any allusion to the compensatory growth and thickening that takes place in bones that have a double duty imposed on them.

Sir James Paget also remarks, "We have seen that when a part is, within certain limits, over-exercised, it is over-nourished; so, if a part be used less than is proper, it suffers atrophy."

Mr. Curling drew attention to the changes which occur in bones after injury, but I cannot find that he refers to the alteration of shape, or increase of growth due to pressure or over-exertion. ${ }^{3}$

The contrast between the injured and sound bones is well illustrated in Cheselden's 'Osteographia.' Mr. Curling adds "that bones as well as soft structures fade and waste away when their activity is diminished or their functions suspended. This is seen in the bones of stumps after amputation, and in bones of anchylosed limbs. In

\footnotetext{
1 ' Lectures on Pathology and Surgery,' 1846, p. 409.

2 'Surgical Pathology,' p. 86, 1863.

3 'Med.-Chir. Trans.,' vol. xx, 1837, p. 341.
} 
the new museum adjoining the Ecole Pratique at Paris, founded by Dupuytren, there is a remarkable skeleton of an adult in which all the bones in the body are anchylosed, excepting the lower jaw and the bones of the shoulderarticulations. The bones of the extremities are very much atrophied, the thigh bones being scarcely larger than an ordinary radius."

When we come to estimate the results likely to be observed after the amputation of a limb through the thighbone we have to consider (1) the function and the action of the muscles, (2) the weight of the body exerting an extra pressure (on the sound side), and (3) the entire removal of all pressure from the stump.

No longer of much use, no longer pressed upon, no longer exercised in proportion to the opposite limb, the whole stump and corresponding side of the pelvis become affected in a somewhat similar manner. On the sound side the bone has to support more than its natural weight, the muscles have to undertake more than their natural duty; the limb in fact has to perform all, if not more than, the work of two legs ; and so bone and muscle are proportionally increased in size, and to some extent altered in shape, while the vascular supply is rendered equal to the demand.

I must again refer to a remark of Mr. Curling's in connection with the rather rapid absorption of bone from non-use. He says in reference to the case quoted from Cheselden, that the late Mr. John Shaw attributed the thinning of the femur to the want of exercise; but adds that " the wasting had taken place to too great an extent, in a short time to be accounted for in this way alone."

I venture to express the opinion that though the wasting of bone under such circumstances as we have considered is necessarily slower than that of muscle, in both it is often more rapid than may be generally supposed. We witness the rapid falling away of muscle in the early stages of hip-joint disease, and had we the power or means to test the waste of bony structure in its commencement, 
and early stages of deterioration, my impression is that we should find the process of absorption, simply as the result of inaction, sufficiently active to account for the changes observed in Cheselden's case.

I further venture to express the opinion, after some little observation, that bone tissue and muscular fibre, under certain circumstances, take on more rapid increase than is often suspected.

In an instance in which the first, second, and fourth fingers were removed by me for an accident, the thumb and remaining third finger soon became so mobile that the mutilated hand might be said to be almost as useful as the original one. Within a few months both thumb and finger were decidedly larger, broader, and longer than the corresponding portions of the fellow hand, and in the course of some year and a half a marked increase in size and length in both had taken place.

We are all aware that the slow changes which are constantly going on in bones from birth till death are regulated and modified, so as not to interfere with the form, substance, and strength of their respective parts, so long as healthy action is permitted, and maintained. But as age advances and movement becomes more limited, bone commences to lose its solidity and becomes more oily and is rendered more brittle.

What, however, appears to me to be the most interesting and important question in connection with the specimens figured in Plate IX is the fact that we find in one and the same subject and at the same time two very distinct conditions in the thigh-bones of the opposite limbs ; two very distinct and different processes, carried on from the time of the amputation of one limb, to the death of the individual who is certified to have lived over eighty years. On the amputated side the remains of the femur are thinned, oily, and brittle. On the sound side the bone is thick, compact, and firm. In the first, motion, and consequently nutrition, have been interfered with, and we witness the progress of decay. In the second, muscular 
action has not only been well preserved, but greatly increased, and here we find the part equal to all the conditions of bone in earlier life.

If such conditions are found to occur under certain known circumstances, is it unreasonable to assume that these facts may, with some slight advantage, be borne in mind in the treatment of certain affections of the osseous system, dependent, not on disease, but on general constitutional disorder? So that, by a careful combination of exercise, position, and rest, combined with the judicious use of mechanical appliances, we may accelerate the improvement of whatever defects such conditions produce.

Subjoined is a short table of specimens illustrative of deterioration of bone, consequent on amputation, paralysis, \&c.

\section{LIST OF SPECIMENS ILLUSTRATIVE OF DETERIORA- TION OF BONE, CONSEQUENT ON AMPUTATION, PARALYSIS, \&C.}

1. S. D., 51, St. Thomas's Hospital Museum.

A right hip-joint, showing complete bong anchylosis; a section has been made through the bones from side to side. Externally the form of the joint is but little, if at all, altered; the margin of the acetabulum may be traced without much difficulty. The cut surfaces show such intricate union that the crusts and cancellous tissues of the bones are continuous, and it is impossible to distinguish their boundaries. The bones are very heavy and their crust is very com. pact and ivory like. The pelvis in this case shows evident thinning of ilium in centre.

\section{S. D., 20, St. Thomas's Hospital Museum.}

An elbow-joint, in which the total destruction of the articular cartilages and partial absorption of the articular end of the humerus had been followed by firm ligamentous anchylosis, more especially between the humerus and ulna. But chronic inflammation, accom. 
panied by growth of irregular bony spiculæ from the ends of the bone, and the repeated formation of abscesses, gave rise to constitutional irritation, sufficiently severe to render amputation necessary. The preparation shows evident wasting of bone from non-use.

\section{S. C., 62, St. Thomas's Hospital Museum.}

Preparation shows obliquity of neck of femur well marked, after amputation through thigh. No history.

\section{S. C., 2, St. Thomas's Hospital Museum.}

Atrophy of humerus after fracture; upper half of bone remarkably atrophied. Cancellous structure of ununited epiphysis of the head is to a great extent removed, and replaced by soft fat. From this point to the middle of the arm the shaft is exceedingly slender, measuring in the thinnest part a quarter of an inch from before backwards, and rather less from side to side; the long circumference and the medullary cavity appear to retain their proportional size. In the lower half the bone has been fractured in three places. There is, however, no osseous union between the fragments; but they are surrounded on the outer side by an adherent periosteum, and thickened and condensed fibrous tissue, which is also prolonged between their extremities, and unites them more or less perfectly to one another. The same kind of tissue is prolonged into their medullary cavities. The fragments are much thicker than either the upper or lower portions of the humerus.

\section{S. C., 51, St. Thomas's Hospital Museum.}

Upper part of femur after amputation. The end of the stump is rounded and for some short distance above this, especially on the posterior aspect, the thickness of the bone is increased by new periosteal deposit. There is well-marked obliquity of neck.

$$
\text { 6. S. C., } 4 \text {. }
$$

Upper part of femur after amputation. The bone gradually tapers towards its lower extremity. Obliquity of neck well marked.

$$
\text { 7. S. C., } 4^{3} \text {. }
$$

Upper part of femur, after amputation, immediately below lesser. trochanter. The section that has been made shows well the atro. phied condition of the bone, and the closed medullary canal. Obliquity of the neck of the femur very marked. 


\section{Spec. 347, Middlesex Hospital Museum.}

A vertical section of the greater part of a left tibia and fibula, with the tarsus and metatarsus, showing extreme atrophy from disease of leg (paralysis?). The compact tissue is reduced to thin shell, and in places perforated by foramina, due to its total conversion into spongy bone. The greatly expanded medullary cavities, in the recent state, were filled with a pinkish-yellow fatty material from the degenerated medulla. The growth of the bones has been retarded, and the tibia and fibula are markedly curved, the convexity being forwards.

\section{Spec. 348, Middlesex Hospital Museum.}

The upper portion of a tibia and fibula from an amputated stump. The bones, especially the fibula, are much atrophied and very light. Their sawn ends are united by bone, and pointed. This was in the case of an adult, as the epiphyses are ossified.

\section{Sp. 349, Middlesex Hospital Museum.}

The bones of a right upper extremity, with scapula and clavicle showing extreme atrophy. All the bones are very light and fragile. The shaft of the humerus is not thicker than the fibula of a boy, and is twisted. The radius and ulna are rounded, and about equal in diameter to a large goose-quill. Both extremities of the humerus and the lower end of the radius have fractured, possibly in removing or mounting the specimen. An apparent deformity of the hand is probably due to the same cause. This case was no doubt that of an adult, as all the epiphysial points are ossified to the shafts of bone.

\section{Series 1, 2, St. Bartholomew's Hospital Museum.}

A scapula and part of a humerus. The arm had been amputated long before death, and through disease the bones are atrophied, but the humerus in a much greater degree than the scapula. The shaft of the humerus bas less than half its natural diameter and tapers to a slender cone, at the end of which is some rough bone. The marks of the attachments of muscles on it are nearly obliterated, and its texture is high and dry. The head of the humerus is flattened and almost entirely absorbed, and there is a corresponding diminution and change of form in the glenoid cavity. 


\section{Series 1, 3, St: Bartholomew's Hospital Museum.}

Sections of a stump of a humerus, exhibiting the results of atrophy from bony disease after amputation. Its sawn end tapers to a cone; the walls of the shaft are less than a pin in thickness, light and dry; and nearly all the osseous part of its cancellous tissue being removed, the medullary tube appears, after maceration, like a smoothwalled cavity.

\section{S. 1, 4, St. Bartholomew's Hospital Museum.}

Pelvis and lower extremities of a young man. All the bones of the right side are atrophied. The several prominences on the right os innominatum are less marked, and its iliac fossa is more shallow than the corresponding parts on the left side. The bones of the right thigh and leg are all shorter, less in circumference, softer, and lighter than those of the left limb. From the hip-joint to the ankle there is a difference of nearly two inches in the length of the limbs. In compensation for this difference the left foot is directed almost vertically, so that in the erect position of the body (in imitation of which the bones are arranged) the extremities of both limbs are at the same level. All the bones of the right foot are slender, small, and soft. The arch of the sole is much increased by the posterior part of the os calcis projecting more than usually downwards. The shaft of the left femur is enlarged by external deposit of new bone. The muscles of the right limb were small and in a state of fatty degeneration. The limb had probably been affected by infantile paralysis.

(For a report of the discussion on this paper, see 'Proceedings of the Royal Medical and Chirurgical Society,' New Series, vol. ii, p. 65.) 


\section{DESCRIPTION OF PLATE IX,}

On the Changes which occur in Bone and Soft Tissues, after amputation of a limb, and from certain other conditions. By George Pollock, F.R.C.S.

Upper portions of two thigh-bones from the same subject. For full description, see p. 276. 
Plate $\mathbb{X}$.

Med .Chir. Trans. Vol . LXIX.

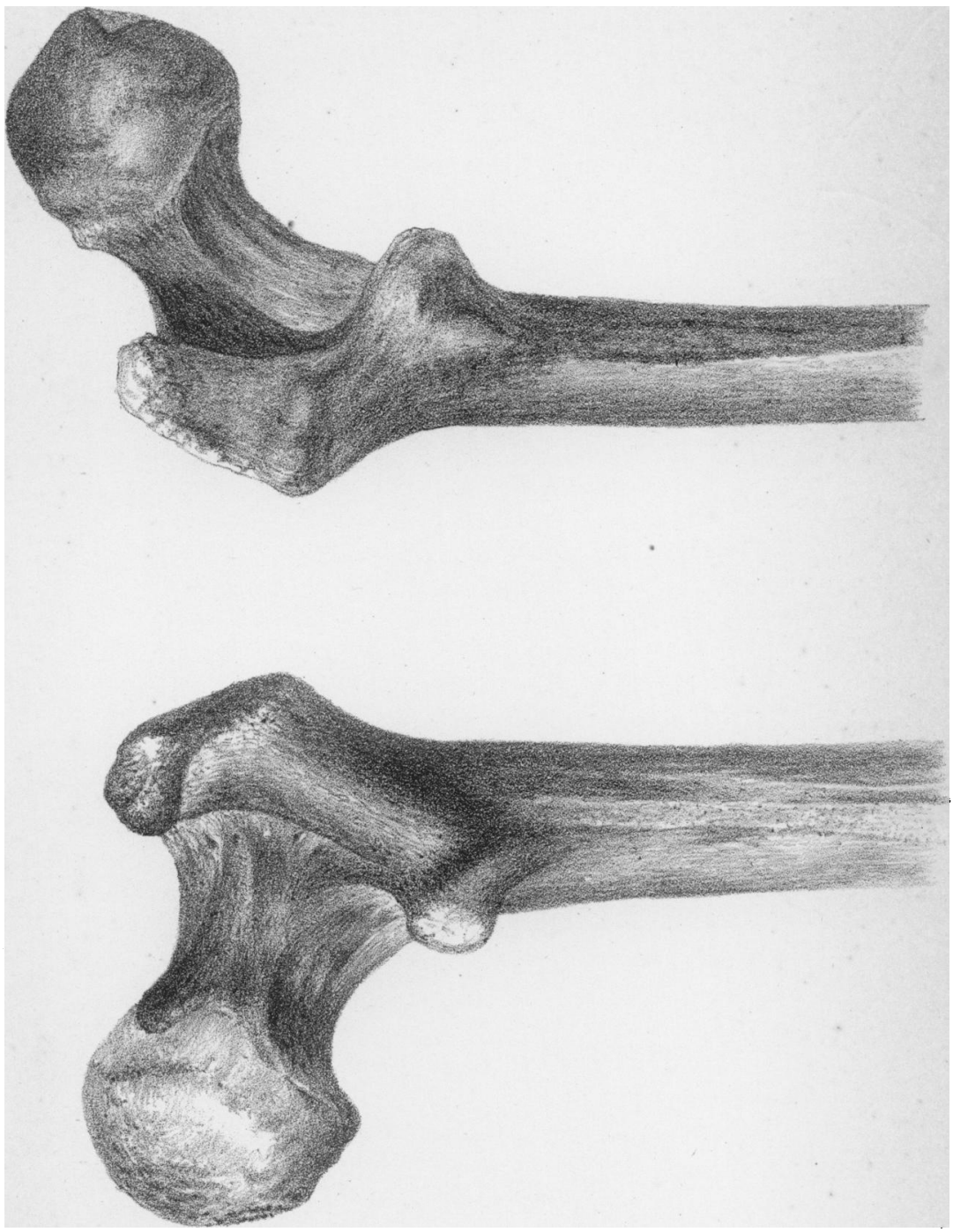

